\title{
COMMUNICATION
}

\section{Ordered self-assembly of proteins for computation in mammalian cells $\dagger$}

Cite this: Chem. Commun., 2014 , 50,676

Received 22nd October 2013, Accepted 4th November 2013

DOI: $10.1039 / c 3 c c 48100 j$

www.rsc.org/chemcomm

\author{
Kui Zhu, $\ddagger^{\mathrm{a}}$ Jianzhong Shen, $\ddagger^{\mathrm{b}}$ Richard Dietrich, ${ }^{a}$ Andrea Didier, ${ }^{a}$ Xingyu Jiang ${ }^{\star c}$ and \\ Erwin Märtlbauer*a
}

A cellular logic system capable of combinatorial and sequential logic operations based on bacterial protein-triggered cytotoxicity was constructed. Advanced devices such as a keypad lock, half-adder and several basic Boolean properties were demonstrated on the cells.

Recent advances in our understanding of biology are critical to the development of future biocomputers, with several distinct advantages, such as flexible design, efficient energy usage, memory functions and error checking. ${ }^{1,2}$ These developed biocomputing systems can be generally grouped into two categories, the bottomup approach uses biomolecules for sensing and performing computation; on the other hand, the top-down method utilizes whole cells including prokaryotic or mammalian cells to implement sophisticated tasks. ${ }^{3-5}$ For example, bacterial virulence proteins have been used as valuable synthetic biology tools to engineer mammalian cells for therapeutic and analytical applications. ${ }^{6}$

Compared to the silicon-based computers using sole electrical signals for information processing, natural cellular biocomputing systems harness various inputs including chemicals, biomolecules and even physical factors such as light and heat. After the extracellular inputs have been sensed by cells, they pass through the first barrier, the cell membrane, then activate downstream effectors in the cytoplasm, and eventually trigger different gene regulators in the nucleus leading to the expression of the corresponding proteins, which will execute specific tasks. ${ }^{7}$ All these events take part in the integral information flow from Input to Output, as shown in Scheme 1a. Whereas the information flow from DNA to RNA and

\footnotetext{
${ }^{a}$ Department of Veterinary Sciences, Ludwig-Maximilians-University Munich, Schönleutnerstr. 8, 85764 Oberschleissheim, Germany.

E-mail: e.maertlbauer@mh.vetmed.uni-muenchen.de, xingyujiang@nanoctr.cn; Fax: +49892180 78576; Tel: +49892180 78602

${ }^{b}$ Department of Pharmacology and Toxicology, College of Veterinary Medicine, China Agricultural University, Beijing 100193, China

${ }^{c}$ CAS Key Lab for Biological Effects of Nanomaterials and Nanosafety,

National Center for NanoScience and Technology, Beijing 100190, China

$\dagger$ Electronic supplementary information (ESI) available: Experimental details and supporting figures and tables. See DOI: $10.1039 / \mathrm{c} 3 \mathrm{cc} 48100 \mathrm{j}$

$\$$ K.Z. and J.S. contributed equally to this work.
}

to protein synthesis, according to the central dogma of molecular biology, is well known, ${ }^{8}$ the crucial role of the cell membrane, the environment-cell interface, in sensing, filtering, amplification, and storage of external signals is still not fully understood. The environment-cell interface shows a phospholipid bilayer architecture to which various receptors and other recognition elements interacting with extracellular stimuli are anchored. ${ }^{9}$ Elucidation of the process of information transduction between Input and the environmentcell interface will facilitate the development of biocomputers with useful human-machine interfaces. In this work, we utilize the selfassembly of a bacterial toxin complex on the cell membrane of immortalized mammalian cells as a 3-input framework to conduct different logic operations (Scheme 1b).

This extracellular protein complex, the nonhemolytic enterotoxin (Nhe), is expressed by most strains of Bacillus cereus, but can also be produced by non- $B$. cereus strains. ${ }^{10}$ The Nhe complex consists of the cytolytic protein NheA and the binding components NheB and NheC. Nhe has some unique properties: (i) the individual Nhe proteins are not toxic, (ii) toxicity can be evoked only by a specific ordered binding sequence, provided that (iii) the Nhe proteins are present in a specific molar ratio, and (iv) Nhe toxicity can be neutralized by antibodies interfering with the binding order.

These properties render the Nhe complex interesting for biocomputing and particularly for constructing Boolean logic gates, which could serve as the basis for sequential logic circuits (SLCs).

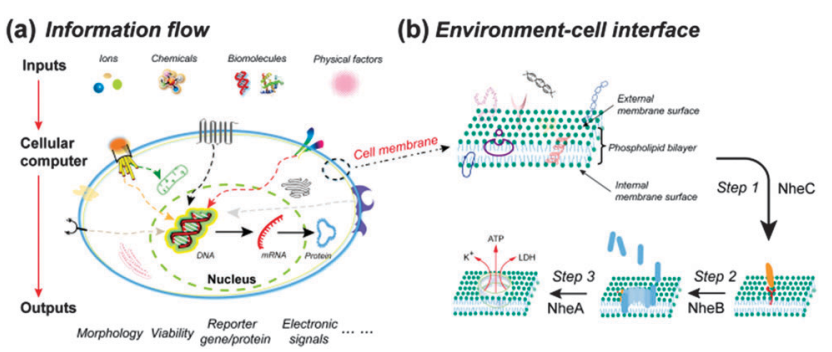

Scheme 1 (a) Schematic representation of a mammalian cell-based biocomputer. (b) The environment-cell interface mediated sequential logic circuit of the Nhe multicomponent toxin complex. 
SLCs are commonly used for the construction of memory devices and storage elements. ${ }^{11}$ Although many logic operations have been achieved in cell-free systems, only a few logic gates have been developed in bacterial and mammalian cells. ${ }^{3-5}$ However, no sequential logic system based on cells has been reported. In this work, we propose a cellular SLC on the natural phospholipid bilayer of the mammalian cell membrane, engineered by the information encoded in the Nhe tripartite protein complex ("Inputs"). In addition, the selfassembly of the ring-shaped nanopore formed by the Nhe complex on the membrane enables multiple readouts for monitoring the dynamic process between the Nhe complex and the cell. We used the developed cellular logic circuit to generate the output signals in this protein-based cellular logic system. ${ }^{6 b}$ We first evaluated the response of several cell lines to the Nhe complex, including A549, Caco-2, HEp-2, IPEC-J2 and Vero cell lines. Vero cells were chosen as a model cell line to perform the following logic operations due to their high susceptibility (Fig. S1, ESI $\dagger$ ). The dynamic response curves of the propidium iodide (PI) and water-soluble tetrazolium salt (WST-1) assays show that the Nhe complex triggers a rapid cellular response in a time-dependent manner (Fig. S2, ESI $\dagger$ ).

We employed the Nhe components as input, and defined the presence and absence of the individual components (NheA, B and C) as a "True" input, or " 1 " and a "False" input, or " 0 ", respectively. The cellular activity of viable cells was regarded as the output, for which we defined a cytotoxicity above $10 \%$ as "True" output, or " 1 " and a cytotoxicity below $10 \%$ as "False" output, or " 0 ". So, we initially constructed a combinatorial AND gate requiring the presence of all three components of the Nhe complex (NheA, B and C =1/1/1), which caused high cytotoxicity to Vero cells (output =1), as shown in Fig. 1 b. Applying a mixture of all three components simultaneously represents a Buffer gate (Fig. 1c). If, however, the Nhe components are added individually to the cell membrane and unbound proteins are removed after each step, an intrinsic binding order is observed (Scheme 1b). The binding order requires that $\mathrm{NheC}$ binds to the cell membrane first (step 1). Together with NheB, which is added in the second step, a stable pre-pore is formed. During this step NheB undergoes a conformational change allowing association of NheA (step 3), which finally leads to cell lysis. ${ }^{12}$ This self-assembly of the three Nhe proteins,

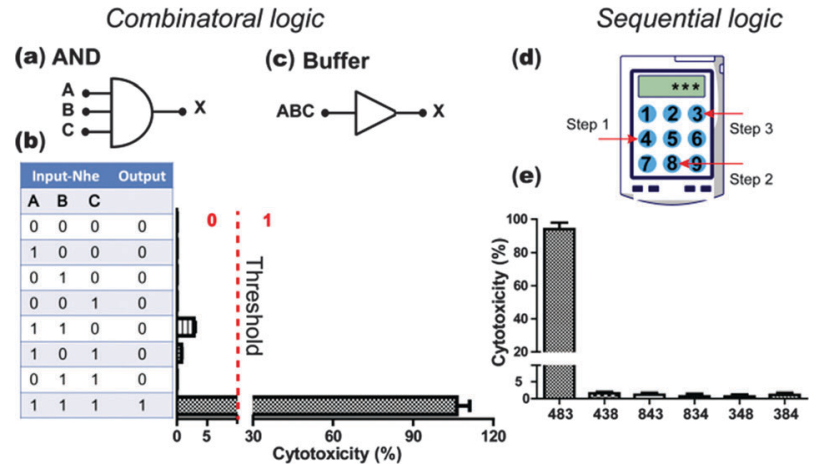

Fig. 1 Nhe-based cellular logic. Equivalent circuit (a) and truth table of the cellular AND gate and the associated cytotoxicity (b). The threshold value ( $10 \%$ of cytotoxicity) is indicated by the dashed red line. (c) Equivalent circuit of a Buffer gate. Scheme (d) and response of the WST-1 assay (e) of all permutations of a three-bit keypad lock. triggered by the contact with the cell membrane, forms a natural model of sequential logic and allows the set-up of an intelligent keypad lock (Fig. 1d and e). As a practical application, we characterized the toxin profiles of $30 \mathrm{~B}$. cereus strains isolated from food samples by using the supernatants of the strains as a Buffer gate (Fig. 1c) together with partial Nhe components (Table S1, ESI $\dagger$ ). All the strains harbored the nhe genes, but some strains did not express the full tripartite Nhe complex at the protein level resulting in a "False (0)" output in the Buffer gate. By supplementing the Buffer negative strains with either NheA and $\mathrm{B}$ (input X2 $=\mathrm{AB}$ ) or NheB and $\mathrm{C}$ (input $\mathrm{X} 2=\mathrm{BC}$ ), the toxin profile could be deciphered.

Based on the recombinant proteins from E. coli (rNheA and rNheC) as well as the mutant strains MHI 1672 (NheA and B) and MHI 1761 (NheB and C), we constructed three "OR" gates for each component of the Nhe complex in the reference strain MHI 1491 (NheA, B and C) (Table S2 and Fig. S3 (ESI †)). To construct INHIBIT gates, physical and biochemical treatments were employed to neutralize the cytotoxic effects of the Nhe complex (Fig. 2a). The Nhe complex is heat-labile ${ }^{13}$ and the cytotoxicity of Nhe is abolished in a temperature-dependent manner. Almost no cytotoxic effect was observed when Nhe was heated at $70{ }^{\circ} \mathrm{C}$ for $10 \mathrm{~min}$. Both NheA and B showed a sharp decrease of cytotoxicity above $50{ }^{\circ} \mathrm{C}$, whereas NheC was heat-stable even at $100{ }^{\circ} \mathrm{C}$ (Fig. S4, ESI $\dagger$ ). A second INHIBIT gate was constructed using a monoclonal antibody (mAb 1E11) against NheB. Addition of mAb 1E11 to the Nhe components nearly completely neutralized the cytotoxic effect caused by the toxin complex (Fig. S5, ESI $\dagger$ ). Disrupting the optimum ratio of the three Nhe components provides yet another means to construct an INHIBIT logic gate. With Nhe the maximum cytotoxicity is achieved when the ratio of the three components is kept at an appropriate level (NheA:B : C = 10:10:1). ${ }^{14}$ Interestingly, as the concentration of NheC increases, the cytotoxicity of the Nhe complex decreases (Fig. S6, ESI $\dagger$ ). Therefore, to obtain a third INHIBIT gate, we just had to add an excess concentration of rNheC to the Nhe complex. (a)

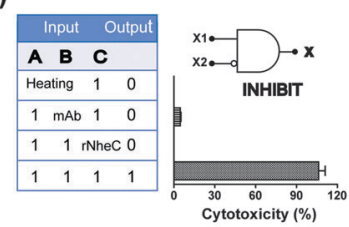

(d)

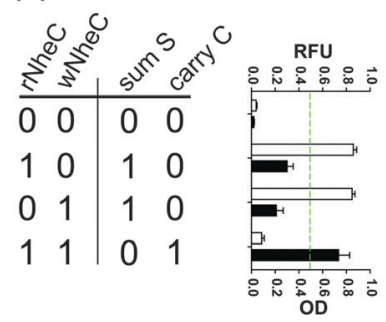

(b)

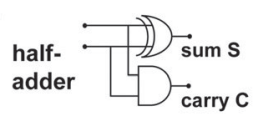

(c)

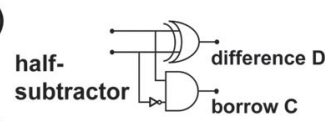

(e)

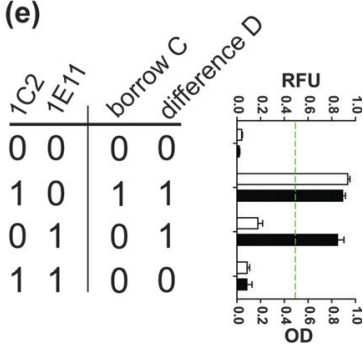

Fig. 2 Equivalent circuit and truth table of the cellular INHIBIT gates and the associated cytotoxicity (a) after applying heat treatment, antibodies, or excess rNheC (X2) to the Nhe complex (X1). Symbolization, truth tables and experimental results of a half-adder (b, d) and a half-subtractor (c, e). OD (absorbance, black bars) and RFU (fluorescence, white bars) represent the results of ELISA and cellular assay. 
(a)

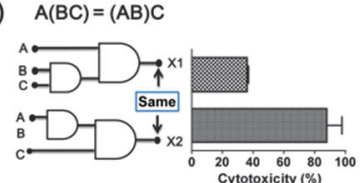

(b) $A+(B+C)=(A+B)+C$

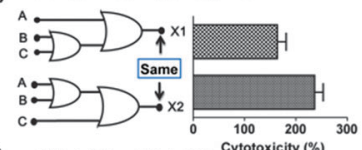

(c)

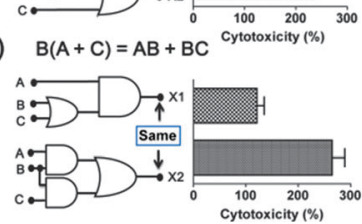

(d)

(e)

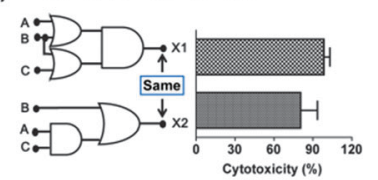

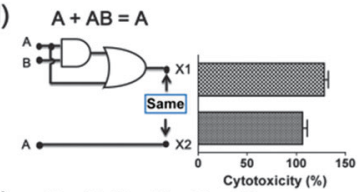

Fig. 3 Cytotoxicity for the associate property of multiplication (a) and addition (b). (a) X1, rNheA plus MHI 1761, and X2, MHI 1672 plus rNheC. (b) X1, MHI 1491 plus rNheA and MHI 1761, and X2, MHI 1491 plus MHI 1672 and rNheC. (c) Cytotoxicity for the distributive property. X1, MHI 1491 plus rNheA and C, X2, MHI 1491 plus MHI 1672 and MHI 1761. Associated cytotoxicity of equation (d) and the simplification of a product-of-sum (e). (d) X1, MHI 1672 plus 1491, and X2, MHI 1491. (e) X1, MHI 1491 plus 1672 and 1761, and X2, MHI 1491 plus NheB, rNheA and C.

Towards a programmable cellular biocomputer, we constructed two commonly used combinational circuits, a halfadder and a half-subtractor, based on the described OR and INHIBIT gates. The half-adder unit performing the addition of two bits, combines a XOR gate with an AND gate (Fig. $2 \mathrm{~b}$ and d). The XOR gate producing a sum $\mathrm{S}$ output is based on the fact that an excess of wild-type NheC (wNheC) and rNheC together with NheB will form a stable NheB-C complex in solution, resulting in no toxicity to cells. It reports a 'False' or ' 0 ' answer, if and only if the presence of inputs is the same $(0+0=0$ and $1+1=10$ ), with 'low' toxicity. In the presence of either input (only one of wNheC and rNheC), it reports 'True' or ' 1 ' answer, with 'high' toxicity. The AND gate producing a carry $\mathrm{C}$ output is based on the readout of an ELISA that specifically detects the concentration of free NheC in solution. Both XOR and AND gates implement their tasks independently and, in parallel, performing precise two-input-two-output computation. In addition, we constructed a half-subtractor device performing the subtraction of inputs requiring the combination of another XOR gate and an INHIBT gate producing difference D and borrow C, Fig. 2c and e. The design of the half-subtractor is based on that two mAbs (1C2 and 1E11) recognize different epitopes of NheB resulting in different reactions in the cytotoxic assay and ELISA. ${ }^{3 b}$ In the XOR gate, either 1C2 or 1E11 reports 'high' values in single-antibody ELISAs, but fails to produce a positive result in the sandwich (double-antibody) ELISA. The INHIBIT gate (1C2 ANDNOT 1E11) is based on the fact that mAb 1E11, but not 1C2, can neutralize toxicity.

Furthermore, examples for the associate property, which applies equally to addition and multiplication, and the distributive property are shown in Fig. 3a-c. Also, two Boolean rules to reduce equations to their simplest forms are presented in Fig. $3 \mathrm{~d}$ and e, the corresponding proofs are shown in Fig. S7 (ESI $\dagger$ ). The ability of performing such fundamental algebraic operations will be essential to simplify logic circuits and to provide diagnostic information.

In conclusion, the use of a multi-component bacterial toxin to control cellular logic gates creates a new input format that could be beneficial for advanced biological computation. The distinct advantages provided by the Nhe-based cellular logic systems allow the setup of a programmable system combining bacterial toxins and cells to perform both combinatorial and sequential logic operations. Moreover, this library of logic gates was used to demonstrate basic Boolean algebraic properties of addition and multiplication (associative and distributive properties) as well as two Boolean rules for simplification. It could be envisioned that this platform can be expanded to include other toxins and proteins to perform sophisticated operations.

We gratefully acknowledge the grants from the German Basic Research Development (973) program (via AiF) and the FEI, Project AiF 17506N, and the Ministry of Science and Technology of China (No. 2013CB127200).

\section{Notes and references}

1 (a) Y. Benenson, Nat. Rev. Genet., 2012, 13, 455; (b) P. Siuti, J. Yazbek and T. K. Lu, Nat. Biotechnol., 2013, 31, 448.

2 (a) E. Katz and V. Privman, Chem. Soc. Rev., 2010, 39, 1835; (b) K. Macvittie, J. Halámek, V. Privman and E. Katz, Chem. Commun., 2013, 49, 6962.

3 (a) A. Prokup, J. Hemphill and A. Deiters, J. Am. Chem. Soc., 2012, 134, 3810; (b) K. Zhu, R. Dietrich, A. Didier, G. Acar and E. Märtlbauer, Chem. Commun., 2013, 49, 9314; (c) E. Katz, J. Wang, M. Privman and J. Halámek, Anal. Chem., 2012, 84, 5463.

4 (a) M. A. TerAvest, Z. Li and L. T. Angenent, Energy Environ. Sci., 2011, 4, 4907; (b) M. A. Arugula, N. Shroff, E. Katz and Z. He, Chem. Commun., 2012, 48, 10174; (c) T. S. Moon, C. Lou, A. Tamsir, B. C. Stanton and C. Voigt, Nature, 2012, 491, 249; (d) Z. Li, M. A. Rosenbaum, A. Venkataraman, T. K. Tam, E. Katz and L. T. Angenent, Chem. Commun., 2011, 47, 3060; (e) A. Tamsir, J. J. Tabor and C. A. Voigt, Nature, 2011, 469, 212.

5 (a) Z. Xie, L. Wroblewska, L. Prochazka, R. Weiss and Y. Benenson, Science, 2011, 333, 1307; (b) W. Weber and M. Fussenegger, Curr. Opin. Chem. Biol., 2011, 15, 414; (c) K. Rinaudo, L. Bleris, R. Maddamsetti, S. Subramanian, R. Weiss and Y. Benenson, Nat. Biotechnol., 2007, 25, 795; (d) S. Ausländer, D. Ausländer, M. Müller, M. Wieland and M. Fussenegger, Nature, 2012, 487, 123.

6 (a) P. Wei, W. W. Wong, J. S. Park, E. E. Corcoran, S. G. Peisajovich, J. J. Onuffer, A. Weiss and W. A. Lim, Nature, 2012, 488, 384; (b) K. Zhu, U. Acaröz and E. Märtlbauer, Chem. Commun., 2013, 49, 5198.

7 (a) K. A. Riccione, R. P. Smith, A. J. Lee and L. You, ACS Synth. Biol., 2012, 1, 389; (b) J. Estrada and R. Guantes, Mol. Biosyst., 2013, 9, 268; (c) T. Pawson, Nature, 1995, 373, 573.

8 (a) I. G. Romero, I. Ruvinsky and Y. Gilad, Nat. Rev. Genet., 2012, 13, 505; (b) K. Chen and N. Rajewsky, Nat. Rev. Genet., 2007, 8, 93. 9 S. W. Hicks and J. E. Galán, Nat. Rev. Microbiol., 2013, 11, 316.

10 (a) L. P. S. Arnesen, A. Fagerlund and P. E. Granum, FEMS Microbiol. Rev., 2008, 32, 579; (b) A. Matarante, F. Baruzzi, P. S. Cocconcelli and M. Morea, Appl. Environ. Microbiol., 2004, 70, 5168.

11 G. de Ruiter, E. Tartakovsky, N. Oded and M. E. van der Boom, Angew. Chem., 2010, 122, 173.

12 T. Lindbäck, S. P. Hardy, R. Dietrich, M. Sødring, A. Didier, M. Moravek, A. Fagerlund, S. Bock, C. Nielsen, M. Casteel, P. E. Granum and E. Märtlbauer, Infect. Immun., 2010, 78, 3813.

13 N. Krause, M. Moravek, R. Dietrich, E. Wehrle, J. Slaghuis and E. Märtlbauer, Int. J. Food Microbiol., 2010, 144, 322.

14 U. Heilkenbrinker, R. Dietrich, A. Didier, K. Zhu, T. Lindbäck, P. E. Granum and E. Märtlbauer, PLoS One, 2013, 8, e63104. 